\title{
The Genetics, Current Research, and Future Treatment of Alport Syndrome
}

\author{
Emily Holland Williams ${ }^{\mathrm{a}}$, Elise Alexandra Kikis ${ }^{\mathrm{a}}$
}

\begin{abstract}
Alport syndrome is a type IV collagen disease that affects the glomerular basement membrane of approximately one in every 5000 people. The disease was first described by A. Cecil Alport in 1927 as "a dominantly inherited hereditary nephritis." The three genotypes of the disease are X-linked dominant, autosomal recessive, and autosomal dominant. The X-linked dominant genotype is the most common, accounting for $80 \%$ of all cases of Alport syndrome, affecting mainly men. The autosomal recessive and autosomal dominant types affect men and women equally. Alport syndrome is caused by mutations on the COLAA3, COL4A4, and COLAA5 genes, which code the $\alpha 3$, $\alpha 4$, and $\alpha 5$ (IV) chains that make up type IV collagen molecules, an important component of basement membranes. Thus, Alport syndrome results in malformed basement membranes, with symptoms including renal impairment, hematuria, bilateral sensorineural hearing loss, and an abnormal structure of the glomerular basement membrane. Alport syndrome also often progresses to end-stage renal disease, especially in men with Xlinked Alport syndrome. At this point, there is no cure for Alport syndrome. However, there are many successful treatments for its symptoms. Angiotensin-converting enzyme (ACE) inhibitors are often given to patients in the early stages of Alport syndrome. For patients with end-stage renal disease, dialysis or kidney transplants are considered the best course of action.
\end{abstract}

Keywords: Alport Syndrome, Genetic Disease, Collagen

\section{Introduction}

Alport syndrome (AS) is a type IV collagen disease first described by A. Cecil Alport in 1927 as "a dominantly inherited hereditary nephritis." 1 The disease is caused by mutations on the COLAA3, COLAA4, or COLAA5 genes. These genes code the $\alpha 3, \alpha 4$, and $\alpha 5$ (IV) chains that make up type IV collagen molecules. ${ }^{2}$ Type IV collagen is an important component of basement membranes as it is necessary to facilitate cell attachment. ${ }^{3}$ Because there are three genes associated with AS, three different mutations can lead to the disease. One mutation is X-linked dominant, another autosomal recessive, and the third is autosomal dominant. The X-linked dominant form of AS (XLAS) accounts for $80 \%$ of cases and affects men much more strongly than women, whereas the autosomal recessive and autosomal dominant cases account for $15 \%$ and $5 \%$ of AS cases, respectively, and affect men and women equally. Symptoms of AS include renal impairment, hematuria, bilateral sensorineural hearing loss, and an abnormal structure of the glomerular basement membrane. Many cases of AS, especially XLAS in males, progress to end-stage renal disease (ESRD). ${ }^{2}$ Though there is currently no cure for AS, treatments include angiotensin-converting enzyme (ACE) inhibitors such as Ramipril for early-stage AS patients, and dialysis or renal transplants for patients with ESRD. ${ }^{4}$

\section{Genetics}

Because AS is heterogeneous having multiple mutations leading to disease, a range of symptoms are observed when comparing different patients. These differences typically relate to hearing ability and the rate of progression for renal failure, while ultra-structural glomerular basement membrane changes occur in each genotype. ${ }^{5}$

XLAS is the most common form. ${ }^{2,6}$ This mutation is located on the long arm of the $\mathrm{X}$ chromosome in the COLAA5 gene. $^{7}$ The COL4A5 gene is made up of approximately $250 \mathrm{~kb}$ of genomic DNA and contains 51 exons encoding a $6.5-\mathrm{kb}$ transcript. ${ }^{8}$ Each family with AS has a different mutation in the COLAA5 gene, and over 700 of these mutations have been described to date.

About $41 \%$ of the mutations in the COLAA5 gene are missense mutations, with about $85 \%$ of these being glycine substitutions in the conserved Gly-Xaa-Yaa repeat of the collagenous domain of the $\alpha 5$ (IV) chain. Another $16 \%$ of mutations are splice site mutations, $22 \%$ are frameshifts, $6 \%$ are nonsense, and the final $15 \%$ are large structural rearrangements. ${ }^{9}$ Mutations in the COLAA5 gene are mostly hereditary, but approximately $15 \%$ of instances of COLAA5 mutations are thought to have occurred de novo without a history of AS in the family. Ninety percent of patients with deletions or nonsense mutations of COLAA5, 70\% of patients with splicing mutations, and $50 \%$ percent of patients with missense mutations will progress to ESRD by age $30 .^{2}$ Deletions may occur anywhere within the COL4A5 gene, or they may encompass the entire gene. ${ }^{7} \mathrm{X}$-linked inheritance typically skips a generation, as there is often an affected female with hematuria but no other distinguishing features of the disease. ${ }^{10}$ As such, she is a carrier of the disease, at risk of passing it to $50 \%$ of her male offspring.

The two other forms of AS are autosomal recessive and autosomal dominant. Mutations on the COLAA3 and COL4A4 genes, located on chromosome 2, are rarer than the X-linked mutations described above and work together to cause both autosomal forms of AS. Autosomal recessive AS is caused by two different COLAA3 mutations or two different COL4A4 mutations in trans, such that each mutation came from a different parent. It is unknown whether there is a genotypephenotype relationship for autosomal recessive AS patients. ${ }^{11}$ About $50 \%$ of mutations on COL4A4 and $45 \%$ of mutations on COLAA3 are missense mutations. Like the missense mutations in COLAA5, $75 \%$ of the missense mutations in COL $4 A 4$ and $85 \%$ in COLAA3 are glycine substitutions in the 
Gly-Xaa-Yaa sequence in the $\alpha 4$ (IV) or $\alpha 3$ (IV) chain respectively. In COLAA4, about $27 \%$ of mutations are frame shifts, $13 \%$ are splice site, $9 \%$ are nonsense, and only about $1 \%$ are larger deletions. In COLAA3, about $21 \%$ of mutations are frame shifts, $15 \%$ are nonsense mutations, $15 \%$ are splice site mutations, and $4 \%$ are larger deletions. There are to date more than 56 known mutations in COLAA4 and 73 mutations in $C O L 4 A 3 .{ }^{9}$

Heterozygous mutations in the COLAA3 or COLAA genes also cause the rare autosomal dominant AS for certain patients. ${ }^{10}$ These dominant mutations can have variations in phenotypes, even between members of the same family carrying the same mutation. ${ }^{12}$

\section{Symptoms}

For a family or individual to be diagnosed as having AS, at least four of the following ten classic criteria must be present: ${ }^{14}$

1. Family history of nephritis or unexpected hematuria in a first degree relative of the initial case, or in a male relative linked through female relatives.

2. Persistent hematuria without another nephropathy, inherited or otherwise, present.

3. Bilateral sensorineural hearing loss in the 2000 to $8000 \mathrm{~Hz}$ range that develops gradually, usually before age 30 .

4. A mutation in the COLAA3, COLAA4, or COL4A5 gene.

5. Immunohistochemical evidence of complete or partial lack of the Alport epitope in glomerular and/or epidermal basement membranes.

6. An abnormal structure of the glomerular basement membrane (GBM), including thickening, thinning, and splitting.

7. Ocular lesions such as anterior lenticonus, posterior subcapsular cataract, posterior polymorphous dystrophy, and retinal flecks.

8. At least two family members with gradual progression to ESRD.

9. Macrothrombocytopenia or granulocytic inclusions.

10. Diffuse leiomyomatosis of the esophagus and/or female genitalia.

Under transmission electron microscopy, glomerular basement membranes in the kidneys include partially thickening, splitting, fragmenting, and basket-weave appearances. ${ }^{15}$ Sensorineural hearing loss is a common symptom, and it primarily affects high tones in AS patients. ${ }^{13}$

However, the presentation of AS differs greatly depending whether the mutation is on the COL4A3, COLAA4, or COLAA5 gene, what type of mutation is present, and whether the patient is male or female. Regarding people with XLAS, male patients always have renal failure while female patients usually do not. About $90 \%$ of male patients develop deafness before age 40 , whereas only about $30 \%$ of female patients ever experience hearing loss, usually between the ages of 30 and 40, and the risk of women becoming fully deaf before age 40 is only $10 \%$. Male patients experience ocular changes (lenticonus, macular fleck, or both) at a rate of $35 \%$, while only $15 \%$ of women do, and most of the changes for women are macular flecks. Hematuria is found in approximately $80 \%$ of male patients and $96 \%$ of female patients, and hematuria is associated with proteinuria in $95 \%$ of male patients and $75 \%$ of female patients. Likewise, female patients generally live into old age whereas male patients historically have died much younger, usually by age 30 but often in their teenage years, though life expectancy is longer now due to advancements in treatment. ${ }^{2,6}$

It is possible to predict the stages of ESRD for men with XLAS by observing older male relatives who are also affected, as the progression of the disease is similar within families. $^{2}$ The progression of XLAS in males can also be predicted by sequencing the genome. For patients with XLAS, genetic testing as a diagnostic tool is accurate at least $90 \%$, so it is highly recommended for men suspected to have AS. ${ }^{9}$ In contrast, it is nearly impossible to predict the progression of the disease for women because there are no statistically significant genotype-phenotype correlations. ${ }^{6}$

Regarding the effect of genotype on XLAS males, patients with nonsense mutations, large rearrangements, or deletion or insertion of base pairs on the COLAA5 gene have a 90\% chance of developing end-stage renal disease before age 30 , and the $50 \%$ renal survival rate for these patients is 32 years. ${ }^{2}$ Likewise, patients with large COL4A5 rearrangements often have severe juvenile AS, usually with deafness. ${ }^{16}$ Indeed, all types of mutations are found in juvenile-type AS, while adult-presenting AS typically only shows glycine substitutions and splicing mutations. ${ }^{8}$ Patients with splice site mutations have a slightly better outcome than those carrying the mutations described above. Specifically, splice site mutations lead to ESRD before 30 in only $70 \%$ of cases; however, the $50 \%$ renal survival rate is 25 years. Men with missense or splice site mutations usually have more than a 20 year age difference for the age of ESRD onset compared to AS patients with large rearrangements or frameshift mutations. For males with large deletions, nonsense mutations, small mutations, and splice site mutations in COL4A5, $50 \%$ will have a hearing defect by age 10 , and men with missense mutations have a $50 \%$ risk of developing a hearing defect by age 20 . Men with a large COL4A5 deletion or point mutation resulting in a premature stop codon also tend to have more lenticonus. Whatever the mutation, though, lenticonus is predictive of early progression to ESRD in affected men. Amino terminal missense mutations are also often associated with ESRD without extrarenal failures. ${ }^{10}$

It is harder to predict the outcome of X-linked AS for women compared to men by gene sequencing, partly because of how rare it is for a woman to be heterozygous for the mutation. Like men, women with missense mutations have the lowest rate of progression to ESRD, but the association is not statistically significant. There is no genotype-phenotype correlation for hearing loss, though the risk of developing ESRD is higher in female patients with hearing loss. There is also no correlation between progression to ESRD before age 31 in female patients and the severity of AS in their relatives with the disease. ${ }^{6}$ Indeed, even if patients do not have hearing deficiencies, ocular lesions, or a family history of AS, doctors should regard AS as a possible diagnosis for patients with hematuria, renal failure, or both because the syndrome can present itself in so many varied ways. ${ }^{13}$

Autosomal recessive AS affects males and females equally often and equally severely. ${ }^{10}$ On a cellular level, in male and female patients with autosomal recessive AS, no $\alpha 3$ $\alpha 4(\mathrm{IV})$ chains are present in capsular and distal tubular 
basement membranes (TBM) while $\alpha 5$ (IV) chains were normal in Bowman's capsules and collecting duct basement membranes. In contrast, in patients with XLAS, $\alpha 3(\mathrm{IV})$, $\alpha 4(\mathrm{IV})$, and $\alpha 5$ (IV) chains are distributed intermittently in females but are absent from the glomerular tuft and extraglomerular BM in males. ${ }^{17}$ The $\alpha 5$ (IV) chain is present in the skin of autosomal recessive patients, though it is not present in X-linked patients. Both males and females present hematuria during infancy, and ESRD can be developed in childhood or adulthood, though full renal failure is uncommon. However, in contrast to XLAS, autosomal recessive AS leads to renal failure usually only in one generation. The exception to this is families with multiple examples of consanguinity, though hematuria may be found in family members. Hearing loss and ocular abnormalities do not occur in patients with autosomal recessive AS. ${ }^{10}$

Autosomal dominant AS is usually much less severe than autosomal recessive or male $\mathrm{X}$-linked AS. The main symptoms of autosomal dominant AS are microhematuria; ESRD onset, usually after age 40; ultrastructural changes of the GBM ranging from isolated thinning to a combination of thinning, thickening, and splitting; and slowly progressive hearing loss in about $20 \%$ of patients. ${ }^{18}$ Retinopathy and cataracts are generally the only ocular abnormalities in autosomal dominant AS patients, but hearing loss and renal impairment have appeared independently within the same family. ${ }^{12}$ There is no genotype-phenotype correlation for autosomal dominant $\mathrm{AS} .^{18}$

\section{Cellular Processes}

The mutations which cause Alport syndrome affect the formation of type IV collagen, a component of many basement membranes. Basement membranes are 300-350 nm thin, non-cellular matrices found in all multicellular animals that separate the epithelium, mesothelium, and endothelium from underlying connective tissue and provide support for the capillary wall. ${ }^{19,3}$ They surround fat cells, individual muscle cells, and Schwann cells, which form myelin by wrapping around peripheral nerve cell axons. ${ }^{3}$ Type IV collagen molecules, laminin networks, entactin, heparan sulfate proteoglycan, and other glycoproteins interact to form basement membranes. ${ }^{7}$ Type IV collagen then interacts with $\alpha 1 \beta 1$ and $\alpha 2 \beta 1$ integrin receptors within basement membranes to facilitate cell attachment to the basement membrane. ${ }^{20}$ Type IV collagen also provides the basement membrane with tensile strength, though it likely does not affect the sizeselectivity or charge-selectivity of the glomerular filter. ${ }^{21}$

There are six identified types of genetically distinct type IV collagen $\alpha$-chains: $\alpha 1$ (IV) through $\alpha 6\left(\right.$ IV). ${ }^{22}$ Type IV collagen consists of three triple-helical proteins made up of the following $\alpha$-chains: $\alpha 1 . \alpha 1 . \alpha 2, \alpha 3 . \alpha 4 . \alpha 5$, and $\alpha 5 . \alpha 5 . \alpha 6 .^{23}$ Each chain is made up of a central collagenous domain of about 1400 amino acid residues containing the repetitive triplet sequence glycine(Gly)-X-Y, with $\mathrm{X}$ and $\mathrm{Y}$ representing other amino acids; a carboxy-terminal noncollagenous (7S) domain of approximately 230 residues; and a noncollagenous amino-terminal sequence of 15 to 20 residues. $^{24,25}$ The $\alpha 5$ chain contains 1685 amino acids, comprised of a 26-residue signal peptide (encoded by exon 1), a 1430-residue collagenous domain (exons 2-47) with 22 short noncollagenous interruptions, and a 229-residue carboxyterminal noncollagenous (NC1) domain (exons 47-51). ${ }^{26}$

The $\alpha 3 . \alpha 4 . \alpha 5$ heterohexamer is made up of one $\alpha 4-\alpha 4$ homodimer and two $\alpha 3-\alpha 5$ heterodimers, for a composition of $(\alpha 3)_{2}(\alpha 4)_{2}(\alpha 5)_{2}{ }^{23}$ The $\alpha 1$ and $\alpha 2$ chains are always present in all basement membranes, and mutations in these genes likely lead to miscarriage. ${ }^{22}$ These chains exist in a $2: 1$ ratio in fetuses, but in adult basement membranes, $\alpha 3, \alpha 4$, and $\alpha 5$ (IV) chains are present in a 1:1:1 ratio. ${ }^{19}$ This is because there is a switch from $\alpha 1$ and $\alpha 2$ (IV) chains to $\alpha 3, \alpha 4$, and $\alpha 5$ (IV) chains during GBM development. ${ }^{27}$ The early stages vesicle, comma, and S-shaped - of GBM development contain only $\alpha 1$ and $\alpha 2$ (IV) chains, while the capillary loop stage contains $\alpha 3, \alpha 4$, and $\alpha 5$ (IV) chains. Indeed, in adults, the $\alpha 3$ and $\alpha 4$ (IV) chains are only expressed in the basement membranes of tissues affected by AS, specifically the glomerular basement membrane, anterior lens capsule, Descemet's membrane, Bruch's membrane, and several basement membranes of the cochlea. ${ }^{7}$

\section{Cellular Malfunctions in AS Patients}

In patients with XLAS, the GBM consists of only $\alpha 1$ (IV) and $\alpha 2$ (IV) membranes. Ultimately, this is because the developmental shift from $\alpha 1$ (IV) and $\alpha 2$ (IV) chains to $\alpha 3$ (IV), $\alpha 4$ (IV), and $\alpha 5$ (IV) chains does not occur, causing the GBM to remain in a fetal state. ${ }^{28}$ Additionally, the $\alpha 3$ (IV) is missing in patients with X-linked AS, and occasionally the $\alpha 4(\mathrm{IV})$, $\alpha 5(\mathrm{IV})$, and $\alpha 6$ (IV) are either absent or abnormal. Missense mutations on the COL4A5 gene tend to result in glycine substitutions in the continuous Gly-X-Y repeats in the $\alpha 5$ (IV) collagenous domain. ${ }^{8}$ This prevents the $\alpha 5(\mathrm{IV})$ chain from folding properly into a triple helix, as glycine, due to its small size, is the only amino acid able that can fit three times in the interior of the tightly wound triple helix. ${ }^{29}$ Nonsense and frameshift mutations on the COL4A5 domain produce a truncated $\alpha 5(\mathrm{IV})$ chain with either no $\mathrm{NC1}$ domain or an $\alpha 5$ (IV) chain with a truncated NC1 domain. ${ }^{8}$ The absence of an $\mathrm{NC} 1$ domain or a nonfunctioning $\mathrm{NC} 1$ domain prevents the formation of the triple-helix and thus the incorporation of the $\alpha 5$ (IV) chain into the basement membrane network. ${ }^{30} \mathrm{~A}$ splice site mutation results in a premature termination of translation or altered form of the protein, obliterating protein function. ${ }^{8}$ Losing the $\alpha 5$ (IV) chain by any mutation causes the loss of the $\alpha 3$ (IV) and $\alpha 4$ (IV) chains as well because the triplehelical $\alpha 3 . \alpha 4 . \alpha 5$ (IV) protomers cannot assemble properly. ${ }^{31}$ For patients with autosomal recessive or autosomal dominant AS, the mechanism that affects the $\alpha 3$ (IV) and $\alpha 4$ (IV) chains is unknown. It is also unknown how exactly GBM thickening, proteinuria, and renal insufficiency occur in AS patients.

\section{Treatments}

As with most diseases, treating AS begins with diagnosing it correctly, and the earlier AS is detected, the better the prognosis. However, since AS shares symptoms with many other renal diseases, and since there are so many AS phenotypes, it is commonly misdiagnosed. In a 2012 study by Yao et al., about $11 \%$ of adolescent and adult AS patients with renal pathology data were previously misdiagnosed: $27.7 \%$ in female XLAS, $17.7 \%$ in autosomal recessive AS, and $6.9 \%$ in male XLAS. Autosomal dominant 
patients were not included in this study because of the small number of cases and the uncertainty of inheritance mode determination. Some of the most common misdiagnoses include thin basement membrane nephropathy, mesangial proliferative glomerulonephritis, and focal segmental glomerulosclerosis. The study found that the best ways to diagnose AS after a misdiagnosis are to determine family history, to re-examine previous biopsy slides, or to perform a new renal biopsy with a complete evaluation of electronic microscopic changes of the GBM and collagen-IV $\alpha$-chain immunofluorescence (COL-IF). ${ }^{32}$ With that being said, in cases where there is renal impairment with a heterozygous COL $4 A 3$ or COL $4 A 4$ mutation, AS should not immediately be diagnosed, as thin basement membrane nephropathy presents similar symptoms. ${ }^{10}$

Since the disease is hereditary, children with a risk of AS should be tested by one year of age, and older people should be tested as soon as there is suspicion of AS. A fast and reliable way to test for XLAS is by skin biopsy specimen analysis because the absence of $\alpha 5$ (IV) chains in the epidermal basement membrane clearly indicates XLAS. ${ }^{30}$ Kidney biopsies may be necessary for patients with autosomal recessive or autosomal dominant AS because $\alpha 5$ chains are found in the epidermal basement membrane of these patients. ${ }^{10}$

Although there are many clinical trials currently underway testing various treatments for AS, for those not involved in these trials, the best solution currently is to annually monitor microalbuminuria and proteinuria by the time children turn one year old, or whenever there is suspicion of AS. Individuals with AS and overt proteinuria should also receive treatment, and treatment should be considered in boys with microalbuminuria for whom there is a high risk of ESRD by age 30 based on mutations in the COL4A5 gene or family history. ${ }^{4}$ Additionally, all AS patients at risk of hearing loss should avoid ototoxic medication and industrial noise exposure to protect their ears. ${ }^{10}$

The most effective treatments for children with AS are those which help delay proteinuria. This is especially important in treating boys with X-linked AS with deletion, nonsense, or splicing mutations, as that genotype leads to the most severe symptoms. Angiotensin-converting enzyme (ACE) inhibition is the recommended first line of therapy because most nephrologists have extensive experience with the therapy and its effects, and ACE inhibitors are easily accessed and inexpensive. ACE inhibitors have been shown to suppress proteinuria, prolong renal function, and increase survival in $\mathrm{COLAA3} 3^{-/-}$mice. Angiotensin receptor blockades (ARB) have also been successful in this regard. ${ }^{4}$ One especially successful ACE inhibitor for AS treatment is ramipril. In a trial using $C O L A A 3^{-/-}$mice, the lifespan of mice treated with ramipril was extended more than $100 \%$ until death by uremia, and the onset of both proteinuria and uremia was delayed. ${ }^{33}$ However, though no side effects of ramipril have been shown, the long-term effects of the drug are unknown and are currently being studied. ${ }^{34}$

Statins - inhibitors of HMG-CoA-reductase used to lower cholesterol - have also proved effective in the treatment of AS via their anti-fibrotic, anti-inflammatory, and antiproliferative effects on certain tissues. Cerivastatin has also been shown to extend the lifespan of $C O L 4 A 3^{-/-}$mice by $28 \%$ while reducing proteinuria and postponing the onset of uremia, regardless of when treatment was begun. Other effects shown in the use of statins include a reduction of both renal fibrosis and inflammatory cell infiltration. ${ }^{35}$

Other treatments that help lower urine protein levels should also be considered, though there is no specific target currently for what these levels should be. Chemokine receptors have been shown to decrease renal fibrosis and tubular and vascular injury in $C O L 4 A 3^{-/-}$mice, suggesting that CCR1 blockade can affect the renal macrophages within the diseased kidney. $^{36}$ Other potential treatments include metalloproteinase inhibitors and stem cell therapy. ${ }^{37,38}$ However, the precise mechanism by which these treatments work is still unknown and is currently being studied.

For patients for whom AS has progressed to ESRD, there are two options: dialysis and renal transplants. Family members with AS should be discouraged from donating kidneys to relatives with AS, but on the chance this occurs, both the donor and recipient should receive nephroprotective treatment from the time of surgery, such as renin-angiotensin system blockade. Considering that $85 \%$ percent of mothers of boys with AS have the mutation and $80 \%$ of these women are diagnosed only after their son or another male relative has been diagnosed, it is important that all family members be genetically tested as soon as possible following initial diagnosis to scan for potential donors. ${ }^{10}$ Though overall renal donations are a safe procedure for AS patients, about $5 \%$ of AS males who receive transplants develop anti-GBM nephritis and lose the allographted kidneys. ${ }^{13}$

Lastly, pregnant women with AS should be monitored during pregnancy, as women with hypertension, proteinuria, or renal impairment are at a higher risk of preeclampsia, and pregnancy may accelerate the decline in renal function. ${ }^{39}$

\section{New research findings}

There are currently several clinical studies underway to study AS progression and treatment. In one study, nine AS adults with albuminuria were treated with a course of benazepril, valsartan, diltiazem, and fluvastatin for four months between 2004 and 2007 after an ACE inhibitor washout period of one month. They then followed Remission Clinic protocol until July 2014. In this trial, median albuminuria declined from $657.7(292.7-1,089.6) \mu \mathrm{g} / \mathrm{min}$ to 71.4 (21.7-504.9) $\mu \mathrm{g} / \mathrm{min}$ during the course of treatment, and raised to 404.3 (167.9-446.8) $\mu \mathrm{g} / \mathrm{min}$ following recovery. Blood pressure and lipids also decreased during the treatment. The treatment also improved the long-term progression of albuminuria for patients who began the trial without renal insufficiency. ${ }^{40}$

Additionally, the EARLY PRO-TECT Alport trial is a double-blind study being conducted in Germany studying the long-term effects of early-onset ramipril therapy on 80 children with $\mathrm{AS}^{34}$ The purpose of this trial is to provide evidence for ramipril treatment for AS, as ramipril is currently being used to treat AS off-label. This trial will also provide information regarding the treatment and maintenance of other rare kidney diseases.

New AS-associated genes are also being identified. For example, Rosado et al. presented findings of a family with autosomal dominant AS in Spain with a new mutation in the COLAA3 gene that causes most of the family members to suffer isolated hearing loss. Interestingly, one family member 
also presents lenticonus, not previously observed in autosomal dominant AS patients. ${ }^{12}$ In another study, 23 total mutations were found in eleven AS patients, each with pathogenic mutations in two collagen IV genes. The researchers also looked for mutations in the extended families of the AS patients, for a total of 56 subjects. This study suggests a digenic inheritance model for AS: “(i) autosomal inheritance with mutations on different chromosomes, resembling recessive inheritance (five families); (ii) autosomal inheritance with mutations on the same chromosome resembling dominant inheritance (two families) and (iii) unlinked autosomal and X-linked inheritance having a peculiar segregation (four families).",41

\section{Concluding Remarks}

AS is caused by mutations in the COLAA3, COLAA4, $A N D$ COLAA5 genes, which code the $\alpha 3, \alpha 4$, and $\alpha 5$ (IV) chains that make up type IV collagen molecules. This results in malformed basement membranes, especially the glomerular basement membrane, with severity dependent on the genotype of the disease. Additional symptoms of AS include renal impairment, hematuria, and bilateral sensorineural hearing loss. In the early stages, AS is often treated with ACE inhibitors, though additional treatments are being studied. AS that has progressed to ESRD is treated with dialysis or renal transplants.

\section{References}

Alport, A. C. Hereditary familial congenital haemorrhagic nephritis. Brit Med J (Clin Res), 1927, 1:504-506.

Jais, J. P., Knebelmann B., Giatras, I., De Marchi, M., Rizzoni, G., Renieri, A., Weber, M., Gross, O., Netzer, K. O., Flinter, F., Pirson, Y., Verellen, C., Weislander, J., Persson, U., Tryggvason, K., Martin, P., Hertz, J.M., Schroder, C., Sanak, M., Krejcova, S., Carvalho, M. F., Suas, J., Antignac, C., Smeets, H., and Gubler, M. C. (2000). X-linked Alport syndrome: Natural history in 195 families and genotype- phenotype correlations in males. $J \mathrm{Am}$ Soc Nephrol, 11, 649-657.

Alberts, B., Johnson, A., Lewis, J., Raff, M., Roberts, K. ,\& Walter, P. (2002). Cell Junctions, Cell Adhesion, and the Extracellular Matrix. In Molecular Biology of the Cell (4th ed., pp. 1164-1169). New York: Garland Science.

Kashtan, C. E., Ding, J., Gregory, M., Gross, O., Heidet,

L., Knebelmann B., Rheault, M., Licht C. (2013). Clinical practice recommendations for the treatment of Alport syndrome: a statement of the Alport Syndrome Research Collaborative. Pediatric Nephrology, 28, 5-11.

Feingold, J., Bois, E., Chompret, A., Broyer, M., Gubler, M. C., Grunfeld, J. P. (1989). Genetic heterogeneity of Alport Syndrome. Kidney Int, 27, 672-677.

Jais, J. P., Knebelmann B., Giatras, I., De Marchi, M., Rizzoni, G., Renieri, A., Weber, M., Gross, O., Netzer, K. O., Flinter, F., Pirson, Y., Dahan, K., Wieslander, J., Persson, U., Tryggvason, K., Martin,
P., Hertz, J. M., Schroder, C., Sanak, M., Carvalho, M. F., Suas, J., Antignac, C., Smeets, H., Gubler, M. C. (2003). X-linked Alport syndrome: Natural history and genotype- phenotype correlations in girls and women belonging to 195 families: a "European community Alport syndrome concerted action" study. J Am Soc Nephrol, 14, 2603-2610.

Kashtan, C. E., Michael, A. F. (1996). Alport syndrome. Kidney Int, 50, 1445-1463.

Knebelmann, B., Breillat, C., Forestier, L., Arrondel, C., Jacassier, D., Giatras, I., Drouot, L., Deschenes, G., Grunfeld JP, Broyer, M., Gubler MC, Antignac C. (1996). Spectrum of mutations in the COL4A5 collagen gene in X-linked Alport syndrome. Am J Hum Genet, 59, 1221-1232.

Hertz, J. M., Thomassen, M., Storey, H., Flinter F. (2012). Clinical utility gene card for Alport syndrome. Eur J Hum Genet, 20, 6.

Savige, J., Gregory, M., Gross, O., Kashtan, C., Ding, J., Flinter F. (2013). Expert guidelines for the management of Alport syndrome and Thin Basement Membrane Nephropathy. J Am Soc Nephrol, 24, 364-375.

Mariyama, M., Zheng, K., Yang-Feng, T. L., Reeders, S. T. (1992). Colocalization of the genes for the $\alpha 3$ (IV) and $\alpha 4$ (IV) chains of type IV collagen to chromosome 2 bands q35-q37. Genomics, 13, 809813.

Rosado, C., Bueno, E., Fraile, P., García-Cosmes, P., \& González-Sarmiento, R. (2015). A new mutation in the COL4A3 gene responsible for autosomal dominant Alport syndrome, which only generates hearing loss in some carriers. Eur J Med Genet, $58.1,35-38$

Tryggvason, K., Heikkila, P., Pettersson, E., Tibell, A., Thorner P. (1997). Can Alport syndrome be treated by gene therapy? Kidney Int, 51, 1493-1499.

Gregory, M. C., Terreros, D. A., Barker, D. F., Fain, P. N., Denison, J. C., Atkin, C. L. (1996). Alport syndrome clinical phenotypes, incidence and pathology. Molecular Pathology and Genetics of Alport Syndrome, 117, 1-28.

Okada, S., Inaga, S., Kitamoto, K., Kawaba, Y., Nakane, H., Naguro, T., Kaidoh, T., Kanzaki S. (2014). Morphological diagnosis of Alport syndrome and thin basement membrane nephropathy by low vacuum scanning electron microscopy. Biomedical Research (Tokyo), 35.5, 345-350.

Antignac, C., Knebelmann, B., Drouot, L., Gros, F., Deschenes, G., Hors-Cayla, M. C., Zhou, J., et al. (1994). Deletions in the COL4A5 collagen gene in $\mathrm{X}$-linked Alport syndrome: characterization of the pathological transcripts in nonrenal cells and correlation with disease expression. J Clin Invest, 93, 1195-1207.

Gubler M. C., Knebelmann B., Beziau, A., Broyer, M., Pirson, Y., Haddoum, F., Kleppel, M. M., Antignac, C. (1995). Autosomal recessive Alport syndrome: Immunohistochemical study of type IV collagen chain distribution. Kidney Int, 47, 11421147.

Marcocci, E., Uliana, V., Bruttini, M., Artuso, R., 
Silengo, M. C., Zerial, M., Bergesio, F., Amoroso, A., Savoldi, S., Pennesi, M., Giachino, D., Rombola, G., Fogazzi, G. B., Rosatelli, C., Martinhago, C. D., Carmellini, M., Mancini, R., Di Costanzo, G., Longo, I., Renieri, A., Mari F. (2006). Autosomal dominant Alport syndrome: molecular analysis of the COL4A4 gene and clinical outcome. Nephrol Dial Transplant, 24, 1464-1471.

Hudson, B. G., Tryggvason, K., Sundaramoorthy, M.,

Neilson, E. G. (2003). Alport's syndrome, Goodpasture's syndrome, and type IV collagen. New England J Med, 348, 2543-2556.

Vandenberg, P., Kern, A., Ries, A., Luckenbill-Edds, L., Mann, K., Kuhn K. (1991). Characterization of a type IV collagen major cell binding site with affinity to the $\alpha 1 \beta 1$ and $\alpha 2 \beta 1$ integrins. $J$ Biol Chem, 113, 1475-1483.

Tryggvason, K., Patrakka, J., Wartiovaara J. (2006). Hereditary proteinuria syndromes and mechanisms of proteinuria. New England J Med, 354, 13871401.

Zhou, J., Reeders, S. T. The $\alpha$ chains of type IV collagen. Molecular Pathology and Genetics of Alport Syndrome. Edited by Tryggvason, K., Basel, Karger, 1996, pp 142-153.

Borza, D. B., Bondar, O., Todd, P., Sundaramoorthy, M., Sado, Y., Ninomiya, Y., Hudson, B. G. (2002). Quaternary organization of the goodpasture autoantigen, the alpha3(IV) collagen chain. Sequestration of two cryptic autoepitopes by intrapromoter interactions with the alpha4 and alpha5 NC1 domains. J Biol Chem, 277, 4007540083.

Leinonen, A., Mariyama, M., Mochizuki, T., Tryggvason, T., Reeders, S. T. (1994). Complete primary structure of the human type IV collagen a4(IV) chain. J Biol Chem, 269, 26172-26177.

Reeders, S. T. (1992). Nephrology Forum: Molecular genetics of hereditary nephritis. Kidney Int, 42, 783-792.

Zhou, J., Leinonen, A., Tryggvason, K. (1994). Structure of the human type IV collagen COL4A5 gene. $J$ Biol Chem, 269, 6608-6614.

Miner, J. H., Sanes, J. R. (1994). Collagen IV alpha3, alpha4, and alpha5 chains in rodent basal laminae: sequence, distribution, association with laminins, and developmental switches. The J Cell Biol, 127, 879-891.

Kalluri, R., Shield, C. F., Todd, P., Hudson, B. G., Neilson, E. G. (1997). Isoform switching of type IV collagen is developmentally arrested in X-linked Alport syndrome leading to increased susceptibility of renal basement membranes to endoproteolysis. $J$ Clin Invest, 99, 2470-2478.

Piez, K. A. Molecular and aggregate structures of the collagens, in Extracellular Matrix Biochemistry, edited by Piez KA, Reddi AH, New York, Elsevier, 1984, pp 1-39.

Van der Loop, F., Monnens, L., Schroder, C., Lemmink, H., Breuning, M., Timmer, E., Smeets H. (1999). Identification of COL4A5 defects in Alport's syndrome by immunohistochemistry of skin. Kidney Int, 55, 1217-1224.

Gunwar, S., Ballester, F., Noelken, M. E., Sado, Y., Ninomiya, Y., Hudson, B. G. (1998). Glomerular basement membrane. Identification of a novel disulfide-cross-linked network of alpha3, alpha4, and alpha5 chains of type IV collagen and its implications for the pathogenesis of Alport syndrome. J Biol Chem, 273, 8767-8775.

Yao, X., Chen, X., Huang, G., Yu, Y., Xu, S., Hu, Y., Wang, Q., Chen, H., Zeng, C., Ji, D., Hu, W., Tang, Z., Liu Z. (2012). Challenge in pathological diagnosis of Alport syndrome: evidence from correction of previous misdiagnosis. Orphanet Journal of Rare Diseases, 7, 100.

Gross, O., Beirowski, B., Koepke, M. L., Kuck, J., Reiner, M., Addicks, K., Smyth, N., SchulzeLohoff, E., \& Weber, M. (2003). Preemptive ramipril therapy delays renal failure and reduces renal fibrosis in COL4A3-knockout mice with Alport syndrome1. Kidney Int, 63.2, 438-446.

Gross, O., Friede, T., Hilgers, R., Görlitz, A., Gavénis, K., Ahmed, R., \& Dürr, U. (2012). Safety and Efficacy of the ACE-Inhibitor Ramipril in Alport Syndrome: The Double-Blind, Randomized, Placebo-Controlled, Multicenter Phase III EARLY PRO-TECT Alport Trial in Pediatric Patients. ISRN Pediatrics, 2012, 436046.

Koepke, M. L., Weber, M., Schulze-Lohoff, E., Beirowski B., Segerer, S., Gross O. (2007). Nephroprotective effect of the HMG-CoA-reductase inhibitor cerivastatin in a mouse model of progressive renal fibrosis in Alport syndrome. Nephrol Dial Transplant, 22, 1062-1069.

Ninichuk, V., Gross, O., Reichel, C., Khandoga, A., Pawar, R. D., Ciubar, R., Segerer, S., Belemezova, E., Radomska, E., Luckow B., Perez de Lema, G., Murphy, P. M., Gao, J. L., Henger, A., Kretzler, M., Horuk, R., Weber, M., Krombach, F., Schlondorff, D., Anders, H. J. (2005). Delayed chemokine receptor 1 blockade prolongs survival in collagen 4A3-deficient mice with Alport disease. J Am Soc Nephrol, 16, 977-985.

Zeisberg, M., Khurana, M., Rao, V. H., Cosgrove, D., Rougier, J. P., Werner, M. C., Shield, C. F., Werb, Z., Kalluri R. (2006). Stage-specific action of matrix metalloproteinases influences progressive hereditary kidney disease. PLOS Medicine, 3, e100.

Sugimoto, H., Mundel, T. M., Sund, M., Xie, L., Cosgrove, D., Kalluri R. (2006). Bone-marrowderived stem cells repair basement membrane collagen defects and reverse genetic kidney disease. PNAS, 103, 7321-7326.

Steele, D. J., Michaels, P. J. (2004). Case records of the Massachusetts General Hospital. Weekly clinicopathological exercises. Case 40-2004- a 42year-old woman with long-standing hematuria. New England J Med, 351, 2851-2859.

Daina, E., Cravedi, P., Alpa, M., Roccatello, D., Gamba, S., Perna, A., Gaspari, F., Remuzzi, G., \& Ruggenenti, P. (2015). A Multidrug, 
Antiproteinuric Approach to Alport Syndrome: A Ten-Year Cohort Study. Nephron.

Mencarelli, M. A., Heidet, L., Storey, H., van Geel, M.,
Knebelmann, B., Fallerini, C., ... \& Renieri, A. (2015). Evidence of digenic inheritance in Alport syndrome. Journal of medical genetics, jmedgenet2014. 